\title{
Near-miss maternal morbidity from severe haemorrhage at caesarean section: A process and structure audit of system deficiencies in South Africa
}

\author{
T S Maswime, ${ }^{1}$ MB ChB, FCOG (SA), MMed, PhD; E Buchmann, ${ }^{2}$ MB BCh, FCOG (SA), MSc, PhD \\ ${ }^{1}$ Wits Obstetrics and Gynaecology Clinical Research Division, Faculty of Health Sciences, University of the Witwatersrand, Johannesburg, \\ South Africa \\ ${ }^{2}$ Department of Obstetrics and Gynaecology, Faculty of Health Sciences, School of Clinical Medicine, University of the Witwatersrand, Johannesburg, \\ South Africa
}

Corresponding author: T S Maswime (tumishang.maswime@wits.ac.za)

\begin{abstract}
Background. A rising caesarean section rate and substandard peri-operative care are believed to be the main reasons for recent increases in maternal deaths from bleeding during and after caesarean section (BDACS) in South Africa (SA). The Donabedian model assumes that clinical outcomes are influenced by healthcare workers and the healthcare system.

Objectives. To evaluate near-miss cases from BDACS with regard to health system structure (resources and facilities) and process (patient care) Methods. A cross-sectional prospective study was conducted in greater Johannesburg, SA. Data of women who had near-miss-related BDACS were collected by means of ongoing surveillance at 13 public hospitals. The World Health Organization intervention criteria were used to identify near-miss cases. A comparison of structure and process between the healthcare facilities was conducted.

Results. Of 20527 caesarean sections, there were 93 near misses and 7 maternal deaths from BDACS. Dominant risk factors for near misses were previous caesarean section (43.9\%), anaemia (25.3\%) and pregnancy-induced hypertension (28.6\%). Eighteen women were transferred to higher levels of care, and 8 (44.4\%) experienced transport delays of $>1$ hour. The caesarean section decision-to-incision interval (DII) was $\geq 60$ minutes in 77 of 86 women, with an average interval of 4 hours. Structural deficiencies were frequently present in district hospitals, and there were serious delays in ambulance transfer and DIIs at all levels of care.

Conclusion. The majority of the women had risk factors for BDACS. There were major ambulance delays and lack of facilities, mostly in district hospitals. All women required life-saving interventions, but could not access appropriate care timeously. Prevention and management of BDACS require a fully functional health system.
\end{abstract}

S Afr Med J 2017;107(11):1005-1009. DOI:10.7196/SAMJ.2017.v107i11.12340

A rising caesarean section rate combined with substandard surgery and postoperative care are believed to be the main reasons for the recent increases in maternal deaths from bleeding during and after caesarean section (BDACS) in South Africa (SA) ${ }^{[1]}$ The confidential enquiries report for maternal deaths in SA for 2011 - 2013 recorded 221 deaths from BDACS. ${ }^{[2]}$ The frequent occurrence of women bleeding to death at or after caesarean section should be unacceptable in any country. Reasons for a rising caesarean section rate in SA are poor observation of women in labour, poor interpretation of cardiotocographic tracings, healthcare worker delays in attending to obstetric emergencies, and a preference by healthcare workers for caesarean section over assisted deliveries. ${ }^{[3]}$

When the quality of healthcare is questioned, as with BDACS, an audit of adverse outcomes may provide information to allow improvements. ${ }^{[4]}$ Maternal death from BDACS rarely happens, thus not allowing an accumulation of cases from which to identify recurring problems. Therefore, auditing near-miss morbidity offers an opportunity to examine enough cases of BDACS to identify healthcare quality issues. Near-miss audits in healthcare have been adopted worldwide for various conditions. ${ }^{[5]}$ Near misses and death share similar characteristics, but the former occurs more frequently and provides information on clinical interventions in survivors. ${ }^{[6]} \mathrm{An}$ audit of maternal near misses offers useful insights into successes and failures in life-threatening obstetric conditions, including BDACS. ${ }^{[7]}$
A useful and validated method of assessing quality of care is the Donabedian model. ${ }^{[4]}$ This model assumes that a clinical outcome is influenced by the healthcare worker (the process of diagnosis and treatment) and the healthcare service structure (the facility and resources where the outcome took place). The model can be applied to circumstances surrounding access to emergency obstetric care. A quality-of-care audit of near misses specifically resulting from BDACS has not been done in SA or globally. The objective of this study was to evaluate maternal near-miss morbidity in terms of process and structure quality-of-care deficiencies related to BDACS.

\section{Methods}

This was a cross-sectional prospective study conducted in southern Gauteng province (greater Johannesburg), SA, from July to December 2014. Approval to conduct the study was obtained from the University of the Witwatersrand Human Research Ethics Committee (ref. no. M140137), and from the Gauteng provincial health department. Gauteng is the most populous and densely populated province in SA, with a rapidly growing urban population. Despite having teaching hospitals with specialised care, the province, and the rest of the country, has been affected by a rise in maternal deaths from BDACS. Eighteen government hospitals in greater Johannesburg offer maternity care, including caesarean section; this study was conducted in 13 of the hospitals -3 tertiary referral, 7 regional and 3 district hospitals. 
At the district hospitals there are no full-time specialists or blood banks on site; patients who have complications are transferred to higher levels of care. The regional hospitals have specialist obstetricians, blood banks and intensive care units (ICUs), but rely on teams of non-specialist doctors to perform the bulk of the clinical and surgical work. The tertiary referral hospitals are university teaching institutions, with a large complement of specialist staff and residents in training. The Johannesburg inner city has 3 tertiary and 2 small district hospitals, with no regional hospitals, resulting in the tertiary hospitals frequently being overburdened with the obstetric workload. About $20 \%$ of births occur in clinics run by midwives, and $5 \%$ occur in women's homes; hence, hospital-based caesarean section rates are higher than the population rates.

The definition of a maternal near miss was based on the World Health Organization (WHO)'s intervention criteria. ${ }^{[5]}$ Inclusion criteria for near-miss morbidity resulting from BDACS are any woman with a gestational age $\geq 24$ weeks or who delivered a neonate $\geq 500 \mathrm{~g}$, undergoing a caesarean section with a combined intraoperative and postoperative blood loss of $\geq 1000 \mathrm{~mL}$, and at least one of the following: blood transfusion $\geq 3 \mathrm{U}$ intraoperatively and/or after completion of the caesarean section; emergency hysterectomy; repeat laparotomy; transfer to a higher level of care; non-anaesthetic postoperative ventilation; use of inotropic drugs; acute dialysis; cardiopulmonary resuscitation; and admission to an ICU as a result of excessive haemorrhage. Women with a ruptured uterus, extra-uterine pregnancy, or severe antepartum bleeding preceding the caesarean section, were excluded. All women meeting the WHO definition during the study period were included. Maternal deaths from BDACS were recorded, but were not audited further.

The main findings of the near-miss audit have been published elsewhere.$^{[8]}$ This article focuses on health system deficiencies that may have led to adverse maternal outcomes. All data were collected by the researcher (TSM), using ongoing surveillance. The study was divided into two parts: (i) a comparison of structure between the hospitals; and (ii) a process audit of near misses and maternal deaths from BDACS. The researcher conducted interviews with the clinical heads of obstetric departments using a questionnaire. The objective of the questionnaire was to determine the facilities at each hospital, such as the presence of a multidisciplinary ICU, a designated obstetric high-care area, an on-site blood bank, the presence of 24-hour operating room facilities and a recovery area (for postoperative observation immediately after surgery), availability of essential drugs (such as uterotonics and tranexamic acid) to prevent or arrest obstetric haemorrhage, and the availability of medical specialists and residents.

Nominated obstetric staff from each hospital were trained to identify a near miss from BDACS; they notified the researcher when cases occurred. Notification was encouraged by regular prompting and visits, including the researcher's attendance at maternal and perinatal review meetings. The researcher screened the identified patients for eligibility according to the WHO definition, and used hospital case records to complete a standard data collection tool. Data collected included antenatal history and events surrounding the admission to hospital, gestational age at first antenatal visit, and number of antenatal visits. Risk factors for BDACS, such as previous caesarean section and antenatal anaemia (haemoglobin $<10 \mathrm{~g} / \mathrm{dL}$ ), were recorded, as were the number of women who were in labour before caesarean section.

Structure was described according to level of care where the caesarean section was performed, the rank of the surgeon who performed the procedure, the most senior surgeon at repeat laparotomy, and documented shortages of ICU beds and blood.
For the quality-of-care audit, process was described according to when patients attended their first antenatal care visit, ambulance delays, delay in initiating the caesarean section (time of decision to operate to start of surgery) and clinical or surgical delays. A first antenatal visit at $\geq 20$ weeks was considered a delay in seeking care ${ }^{[9]}$ The ambulance waiting time was calculated from the time of calling for an ambulance to the time that the patient arrived at the referral hospital. The national standard for ambulance waiting time is $<1$ hour. ${ }^{[10]}$ The international standard for decision-to-incision interval (DII) for caesarean section is 30 minutes; the SA national guidelines, however, accept 60 minutes. ${ }^{[11]}$ Oxytocin is administered routinely to all SA patients during the third stage of labour, regardless of mode of delivery. ${ }^{[1]}$ Clinical and surgical delays were described as any healthcare worker factors that could have caused a delay in patients receiving appropriate management. The number of women with surgical trauma and atonic uterus was also assessed.

A sample of 100 near-miss cases and maternal deaths was envisaged to provide reasonable confidence intervals (CIs) for observed percentage frequencies. With a sample of 100 patients, percentage estimates have $95 \%$ CIs that are not $>10 \%$ above and below the observed point estimated. Data analysis employed quantitative techniques, using Stata 11 software (Statacorp; USA). Descriptive data were analysed using medians, ranges and interquartile ranges for continuous variables, and proportions with percentages for categorical variables. Comparisons of categorical variable frequencies were made using Fisher's exact test.

\section{Results}

The study was conducted in 13 hospitals. Initially 15 hospitals consented to being part of the study, but 2 of these could not provide healthcare workers to do active surveillance. The tertiary hospitals were prompted and/or visited weekly for cases, and the regional and district hospitals at least monthly. The 13 hospitals had 9 blood banks (69.2\%), 8 multidisciplinary ICUs (61.5\%) and 9 obstetric high-care areas (69.2\%). Eleven hospitals (84.6\%) had at least one specialist obstetrician and gynaecologist available in case of emergencies. Four hospitals (30.8\%) were staffed with residents in training. At 2 hospitals, the operating room was not available on all days of the week (Table 1).

Ninety-three women fitted the case definition for near-miss morbidity related to BDACS. Seven maternal deaths related to BDACS were not included among the 93 near-miss cases. The total number of deliveries at the hospitals was 46775 , with 20527 caesarean sections (43.9\%). Frequent risk factors were previous caesarean section in 44 women (47.0\%), antenatal anaemia (21/83 with haemoglobin results; 25.3\%), pregnancy-induced hypertension (26/91; $28.6 \%)$, placenta praevia $(7 / 93 ; 7.5 \%)$ and abruptio placentae $(21 / 93$; $22.6 \%)$. Forty-four women (47.3\%) were in the first stage of labour and $8(8.6 \%)$ in the second stage, while $41(44.1 \%)$ were not in labour. Twenty-seven (29.0\%) women bled from surgical trauma, and 40 $(40.3 \%)$ suffered from atonic uterus.

Interventions used as near-miss markers were blood transfusion $\geq 3 \mathrm{U}$ in 64 women $(68.8 \%)$, repeat laparotomy in 43 (46.2\%), artificial ventilation in 39 (41.9\%), hysterectomy in 38 (40.9\%), ICU admission in $30(32.2 \%)$, transfer to higher level of care in 18 (19.3\%), and acute dialysis in $6(6.5 \%)$.

A majority of the women had attended an antenatal clinic $(n=83 ; 89.2 \%)-13(13.9 \%)$ for the first time at $\geq 20$ weeks of gestation (Table 2). Seven caesarean sections were performed in district hospitals (7.5\%), 22 in regional hospitals (23.7\%) and 64 in tertiary hospitals $(68.8 \%)$. Most of the caesarean sections were initiated by registrars 
Table 1. Available drugs, facilities and human resources by hospital level of care

\begin{tabular}{|c|c|c|c|}
\hline & District hospitals $(n=3)$ & Regional hospitals $(n=7)$ & Central hospitals $(n=3)$ \\
\hline \multicolumn{4}{|l|}{ Available drugs, $n$ (\%) } \\
\hline Oxytocin & $3(100)$ & $7(100)$ & $3(100)$ \\
\hline Ergometrine & $3(100)$ & $3(42.9)$ & $3(100)$ \\
\hline Tranexamic acid injection & $2(66.7)$ & $7(100)$ & $3(100)$ \\
\hline Prostaglandin $\mathrm{F}_{\text {2-alpha }}$ & 0 & $1(14.3)$ & $3(100)$ \\
\hline \multicolumn{4}{|l|}{ On-site facilities } \\
\hline Blood bank & 0 & $6(85.7)$ & $3(100)$ \\
\hline High-care unit & 0 & $6(85.7)$ & $3(100)$ \\
\hline 24-hour postoperative recovery area & $1(33.3)$ & $5(71.4)$ & $3(100)$ \\
\hline 24-hour operating room & $2(66.7)$ & $6(85.7)$ & $3(100)$ \\
\hline Intensive care unit & 0 & $6(85.7)$ & $2(66.7)$ \\
\hline Early-warning charts & $1(33.3)$ & $4(57.1)$ & 0 \\
\hline \multicolumn{4}{|l|}{ Human resources } \\
\hline Specialist obstetricians & $2(66.7)$ & $7(100)$ & $3(100)$ \\
\hline Specialist anaesthesiologists & 0 & $6(85.7)$ & $3(100)$ \\
\hline Specialist paediatricians & 0 & $6(85.7)$ & $3(100)$ \\
\hline
\end{tabular}

Table 2. Process indicators, possibly associated with near misses from bleeding during or after caesarean section

\begin{tabular}{ll}
\hline Indicators & $\boldsymbol{n}(\%)$ \\
\hline Did not attend antenatal clinic $(n=93)$ & $10(10.7)$ \\
First antenatal visit at $\geq 20$ wks $(n=83)$ & $13(15.7)$ \\
Ambulance delays $>1 \mathrm{~h}(n=18)$ & $8(44.4)$ \\
Caesarean section decision-to-incision time & $77(89.5)$ \\
$\geq 60$ min $(n=86)$ &
\end{tabular}

Table 3. Time interval between decision to perform caesarean section $^{*}$ and start of operation by hospital level of care

\begin{tabular}{llll}
\hline Time interval, $\mathbf{h}$ & $\begin{array}{l}\text { District } \\
\text { hospitals } \\
(\boldsymbol{n}=\mathbf{3})\end{array}$ & $\begin{array}{l}\text { Regional } \\
\text { hospitals } \\
(\boldsymbol{n}=7)\end{array}$ & $\begin{array}{l}\text { Tertiary } \\
\text { hospitals } \\
(\boldsymbol{n}=\mathbf{3})\end{array}$ \\
\hline$<1$ & 2 & 3 & 4 \\
1 & 2 & 3 & 16 \\
2 & 2 & 1 & 10 \\
3 & 0 & 3 & 4 \\
4 & 0 & 3 & 12 \\
5 & 0 & 1 & 6 \\
$\geq 6$ & 0 & 5 & 9 \\
Total & 6 & 19 & 61 \\
${ }^{*}$ Caesarean section where time of decision was noted $(n=86)$. &
\end{tabular}

(54/93; 58.1\%) and non-specialist medical officers (29/93; 31.2\%). A specialist assisted during the caesarean section in 23 cases $(24.7 \%)$. At the 43 repeat laparotomies, the most senior surgeons were specialists $(n=23 ; 53.5 \%)$, registrars $(n=11 ; 25.6 \%)$ and non-specialist doctors $(n=7 ; 16.3 \%)$, with the ranks of 2 surgeons unknown. Two women could not be treated in the ICU in their hospital, because of the shortage of beds, even though they met the local eligibility criteria. One woman could not receive blood because of shortages at the blood bank. Of 18 women transferred to a higher level of care, 8 (44.4\%) experienced ambulance delays of $>1$ hour. One woman waited for a little more than 8 hours. Seventy-seven of 86 women in whom the time of decision to operate was noted (89.5\%), had a DII for caesarean section $\geq 1$ hour, with the longest waiting times being 21 hours and 42 hours. Over half $(44 / 86 ; 51.0 \%)$ of the caesarean sections were done $\geq 4$ hours after the decision to operate (Table 3 ). The median DII was 4 completed hours in both regional $(n=19)$ and tertiary $(n=61)$ hospitals.

Seven women died from BDACS. Three died suddenly and unexpectedly before the cause of bleeding was established. One died as a result of a previous placenta accreta during a caesarean section. Two women had abruptio placentae with intraoperative haemorrhage from uterine atony, and 1 woman bled excessively from surgical trauma. Five of the 7 women had no surgical risk factors, and all had blood loss $(\leq 700 \mathrm{~mL})$ at caesarean section.

\section{Discussion}

This study found serious delays with regard to ambulance transfer and excessively long DIIs. Structural deficiencies were especially frequent at district hospitals. Many women had risk factors for BDACS prior to caesarean section. All the women in this study required life-saving interventions, which could not be performed at all the hospitals. Successful treatment of complications from BDACS requires a well-resourced, functional health system, skilled healthcare workers and an effective inter-hospital emergency transport system.

Maternal deaths in Africa are associated with poor access to healthcare, shortage of skilled healthcare workers, and lack of resources. ${ }^{[12]}$ While our study identified a number of system deficiencies and delays, maternal deaths were uncommon, suggesting that the health system is largely intact and able to prevent most maternal deaths from BDACS.

Antenatal care can contribute to preventing difficulties at caesarean section. In SA, every woman should be given a delivery plan, including the expected date, place and mode of delivery. ${ }^{[13]}$ Early booking allows women with risk factors for BDACS to be identified and delivered at appropriate centres. A majority of the women started attending the antenatal clinic early in their pregnancy. Previous caesarean section is an obvious example, being associated with abdominal adhesions, placenta praevia and morbidly adherent placenta. Current operative obstetrical procedures are challenged by the increasing caesarean section rate, associated to a large extent with greater numbers of women who have had previous caesarean sections, and the attendant surgical difficulties. ${ }^{[14]}$

There are various classifications for the timing of caesarean section according to urgency. The 30-minute rule for the DII lacks scientific 
evidence, and is regarded as such by experts, as it is not supported by clinical trials. ${ }^{[15]}$ Several studies of maternal outcomes of a prolonged DII did not find an increase in endometritis, wound infection, operative injuries or caesarean hysterectomy. ${ }^{[16,17]}$ Thomas et al. ${ }^{[18]}$ however, found that an interval of $>75$ minutes was associated with an increased number of women requiring special care. A study done in Malawi had an average DII of 1.69 hours. ${ }^{[19]}$ Lack of operating room space and high patient volume contributed to delays. The average DII in this study was 4 hours, which seems excessive. Long delays most frequently occurred at tertiary hospitals, suggesting that these services are overloaded, possibly associated with a lack of capacity at the two district hospitals in Johannesburg. This is far longer than even the generous 75 minutes recommended in the NICE guidelines for caesarean section that is not necessarily life threatening. ${ }^{[20]}$ Factors affecting the DII include clinicians' perceptions of urgency, shortage of staff, operating room delays, delays due to anaesthetic complications, and staff not being on site. ${ }^{[15]}$ Some of these factors may be associated with preoperative neglect of women awaiting caesarean section, putting them at risk of bleeding and/or bleeding complications.

Delay in reaching the appropriate health facility may have played a causal role in BDACS in women transferred to a higher level of care. The shortage of ambulances and appropriate referral systems is a major concern in SA. As shown in this study, delays frequently occurred. Transport of postoperative caesarean section patients with BDACS deserves special priority, because a delay may result in exsanguination while waiting for transfer or treatment at the referral hospital. ${ }^{[2]}$ In light of the number of maternal deaths from BDACS in 2012, Gauteng province increased the number of dedicated obstetric ambulances in 2014. ${ }^{[21]}$ However, the province is still far from reaching the national target for the number of emergency vehicles in the province. ${ }^{[22]}$ The SA confidential enquiries into maternal deaths found that of the 221 deaths from BDACS in 2011 - 2013, 38\% occurred in district hospitals and $43 \%$ in regional hospitals. Delays in transfer to higher levels of care were experienced by 42 women. Delays in inter-institutional transport were reported in $12.2 \%$ of deaths, and many women died in the ambulance before, during or after transfer from a district hospital. ${ }^{[2]} \mathrm{A}$ related issue is periodic non-availability of 24-hour caesarean section facilities in hospitals because of staff shortages, as shown in two of the institutions in this study. Such shortages are unacceptable in an environment where the emergency transport supply is erratic, and also contribute to overloading of regional and tertiary hospitals, leading to excessive waiting times for emergency surgery.

\section{Strengths and limitations of the study}

Strengths of this study include that a relatively large number of near misses from BDACS in a variety of hospitals with different levels of care could be reviewed, which have not been audited before. Limitations include small numbers of maternal deaths, thus not allowing feasible statistical comparison between near misses and maternal deaths in terms of deficiencies in process and structure. Another limitation was the lack of a control group with a good outcome; hence, it could not be ascertained whether the risk factors were unique to women who had life-threatening bleeding. The available data, therefore, do not necessarily allow a causal link to be established between system deficiencies and near misses. Nevertheless, it can reasonably be expected that such deficiencies may lead to bleeding and other complications, resulting in a near miss or death. It was not possible for the researcher to be on site at all the hospitals to ensure comprehensive recruitment of cases.
Prompting was frequent and reporting was possibly complete in tertiary and district hospitals, where near misses were exceptional events. It is likely that there was under-reporting of near-miss cases in the regional hospitals, which were at times prompted only monthly. However, the total number of maternal deaths is considered to be accurate. The study did not have a control group of caesarean section cases that were not near misses. Such a group would have contributed to establishing risk factors and causal roles for system deficiencies, such as prolonged DII. Suggestions for future studies include the effects of prolonged DII on maternal near misses resulting from caesarean section. Quality improvement efforts with regard to access and safety at caesarean section should start with a regular audit of near misses from BDACS at all hospitals that perform caesarean sections. Recommendations for practice would include defining minimal safety criteria for obstetric units that would prevent severe morbidity and maternal death at caesarean section, e.g. staff cover, drugs, operating room availability, and systems to manage caesarean section queues.

\section{Conclusions}

The majority of the women had risk factors for BDACS. There were major ambulance delays, and prolonged decision to caesarean section incision intervals. All women required life-saving interventions, but could not access appropriate care timeously in some of the hospitals. Prevention and management of BDACS require a functional health system with skilled healthcare workers and an effective inter-hospital transport system.

\section{Acknowledgements. None.}

Author contributions. TSM: substantial contributions to the conception and design, acquisition of data, interpretation of data, and drafting of the manuscript, revising it. EB: substantial contributions to the conception and design, analysis, and interpretation of data. TSM and EB: final approval of the version to be published.

Funding. The research was supported by the Carnegie Corporation of New York, Grant B8749.R01, and the South African Medical Research Council, Self-initiated Research Grant.

Conflicts of interest. None.

1. Gebhart G, Fawcus S, Moodley J, et al. Maternal death and caesarean section in South Africa: Results from 2011- 2013 Saving Mothers Report of the National Committee for Confidential Enquiries into Maternal Deaths. S Afr Med J 2015;105(4):287-291. https://doi.org/10.7196/SAMJ.9351

2. Department of Health. Saving Mothers 2011-2013: Sixth Report on Confidential Enquiries into 2. Department of Health. Saving Mothers 2011-2013:
Maternal Deaths in South Africa. Pretoria: DoH, 2015.

Maternal Deaths in South Africa. Pretoria: DoH, 2015.
3. Massyn N, Day C, Peer N, et al. District Health Barometer 2013/14. Durban: Health Sys tems Trust, 2014.

3. Massyn N, Day C, Peer N, et al. District Health Barometer 2013/14. Durban: Health Sys tems Trust, 2014.
4. Donabedian A. Evaluating the quality of medical care. Milbank Q 2005;8(3)3:691-729. https://doi. Donabedian A. Evalu
org $/ 10.2307 / 3348969$

5. Say L, Souza J, Pattinson R. Maternal near miss - towards a standard tool for monitoring quality of maternal health care. Best Pract Res Clin Obstet Gynaecol 2009;23(3):287-296. https://doi. org/10.1016/j.bpobgyn.2009.01.007

6. Pattinson RC, Buchmann E, Mantel G. Can enquiries into severe acute maternal morbidity act as a surrogate for maternal death enquiries? Br J Obstet Gynaecol 2003;110(10):889-893. https://doi. org/10.1111/j.1471-0528.2003.03044.x

7. Penney G, Brace V. Nearmiss audit in obstetrics. Curr Opin Obstet Gynaecol 2007;19(2):145-150. https://doi.org/10.1097/GCO.0b013e328014a860

8. Maswime S, Buchmann E. Why women bleed and how they are saved: A cross-sectional study of caesarean section near-miss morbidity. BMC Pregnancy Childbirth 2017;17(1):15. https://doi.

org/10.1186/s12884-016-1182-7
9. Black V, Solarin I. 'They told me to come back': Women's antenatal care booking experience in innercity Johannesburg. Matern Child Health J 2013;17(2):359-367. https://doi.org/10.1007/s10995-012019-6

10. Marcus J, Clow S. Response times of ambulances to calls from midwife obstetric units of the Peninsula Maternal and Neonatal Service (PMNS) in Cape Town. Curationis 2009;32(1):59-66. https://doi. org/10.4102/curationis.v32i1.886

11. Department of Health. Guidelines for Maternity Care in South Africa. A Manual for Clinics, Community Health Centres and District Hospitals. Pretoria: DoH, 2007.

12. World Health Organization. Maternal Mortality Fact Sheet. Geneva: WHO, 2014

13. Department of Health. Guidelines for Maternity Care in South Africa. Pretoria: DoH, 2015

14. Bonney E, Myers J. Caesarean section: Techniques and complications. Obstet Gynaecol Reprod Med 2011;21(4):97-102. https://doi.org/10.1016/j.ogrm.2010.12.002

5. Yeung T, Lao T. Timing of caesarean section according to urgency. Best Pract Res Clin Obstet Gynaecol 2013;27(2):251-267. https://doi.org/10.1016/j.bpobgyn.2012.09.005 
16. Bloom S, Leveno K, Spong C, et al. Decision-to-incision times and maternal and infant outcomes. Obstet Gynaecol 2006;108(1):6-11. https://doi.org/10.1097/01.AOG.0000224693.07785.14

7. Nasrallah F, Harirah H, Vadhera R, et al. The 30-minute decision-to-incision interval for emergency caesarean delivery: Fact or fiction? Am I Perinatol 2004:21(2):63-68. https.//doi: org/10.1055/s-2004-820513

18. Thomas J, Paranjothy S, James D. National cross sectional survey to determine whether the decision to incision interval is critical in emergency caesarean section. BMJ 2004;328(7441):665. https://doi. org/10.1136/bmj. $38031.775845 .7 \mathrm{C}$

19. Harfouche M, Hosseinipour M, Kaliti S, et al. Quality indicators and outcomes of emergency caesarean deliveries at a district-level maternity hospital. Afr J Reprod Health 2015;19(3):61-67.

20. National Institute for Health and Care Excellence. Caesarean Section Clinical Guideline. 2011. https:// www.nice.org.uk/guidance/cg132 (accessed 19 September 2017).
21. Times Live. Mums-to-be get their own ambulances, 27 February, 2014. http://www.timeslive.co.za/ thetimes/2014/02/27/mums-to-be-get-their-own-ambulances (accessed 20 June 2017).

22. Health-e News. Gauteng the latest province to admit ambulance shortages, 9 June 2015 . http://www. health-e.org.za/2015/06/09/gauteng-the-latest-province-to-admit-ambulance-shortages/ (accessed 20 June 2017)

Accepted 3 July 2017 